\title{
Gestión tecnológica del conocimiento: herramienta moderna para la gerencia de instituciones educativas
}

\section{Technological management of knowledge: modern tool for management of educational institutions}

\author{
William Alejandro Niebles-Núñez ${ }^{1}$ \\ Hugo G. Hernández-Palma² \\ Diego Cardona-Arbeláez ${ }^{3}$.
}

Recibido: diciembre 17 de 2015

Aceptado: junio 28 de 2016

\begin{abstract}
Resumen
El propósito del presente artículo es el de exponer una reflexión teórica orientada a analizar la gestión tecnológica del conocimiento, como herramienta moderna para la gerencia de las organizaciones educativas. Se trata de un estudio de carácter descriptivo, en el que se utilizó la observación bibliográfica a través del fichaje, como técnica para la recolección de información. Entre los hallazgos se tiene que las estrategias más empleadas para la gestión del conocimiento, aunque no muy estables, son: la utilización de espacios colaborativos, los sistemas de recuperación de información, la gestión y utilización de expertos, así como los sistemas basados en conocimiento; en contraste, son escasas las técnicas de gestión documental. Entre las recomendaciones se plantea desarrollar actividades de dinámicas con los docentes, de forma que se consolide el conocimiento de las emociones que conduzcan a un mayor optimismo y motivación.
\end{abstract}

Palabras clave: gestión de conocimiento, instituciones educativas, gerencia moderna.

\begin{abstract}
The aim of this article is to present a theoretical reflection directed to analyze technological management of knowledge as a modern tool for administration of educational organizations. It was framed as a descriptive theoretical reflection. It was used bibliographic observation through the signing as technique. Among reflections, it was found that the strategies used, although not very stable, were: use of collaborative spaces, systems of information retrieval, management, and use of experts, as well as knowledge-based systems. However, document management techniques were scarce. Finally, as recommendation, developing dynamic activities between groups of teachers is proposed, in this way, the knowledge of emotions that leads to greater optimism and motivation can be consolidated.
\end{abstract}

Keywords: knowledge management, education, modern management.

\footnotetext{
1 Administrador de Empresas, Doctor en Dirección Estratégica, Universidad del Atlántico, Colombia. E-mail: williamniebles@yahoo.com.mx

2 Ingeniero Industrial, Magíster en Sistema de Gestión, Universidad del Atlántico, Colombia. E-mail: hugoghernandezpalma@gmail.com

3 Administrador de Empresas, Magister en Desarrollo Empresarial, Universidad Libre Sede Cartagena, Colombia.

E-mail: diego.cardona@unilibrectg.edu.co
} 


\section{Introducción}

Desde hace algunas décadas, en muchos países se vienen implementando estrategias pedagógicas que permiten que, desde el inicio de la edad escolar, se gestione el conocimiento para así desarrollar habilidades sociales que permitan un aprendizaje basado en la retroalimentación con el entorno (Novoa-Ruiz, 2013). El proceso de gestión de conocimiento se ha venido implementando en la mayoría de los países, lo que ha permitido a las entidades educativas, estructurar sus planes de estudio e implementar estrategias que vayan de la mano con las Nuevas Tecnologías de la Información y la Comunicación, NTIC, para que se desarrollen habilidades sociales donde se interactúe con todos los grupos de interés (Cabarcas-Álvarez, 2012; Garcés-Pretel, Ruiz-Cantillo, \& MartínezÁvila, 2014).

Son muchas las investigaciones y apreciaciones acerca de la gestión del conocimiento, así como también de las habilidades sociales que les permiten a los individuos interactuar al ritmo de la tecnología a través de propuestas didácticas y dinámicas en el aula para un aprendizaje más significativo (Castellanos-Niño, 2012; Torres-Ortiz \& Duarte, 2016). En este orden de ideas, se infiere que generar conocimiento no se ciñe solamente a la idea de certeza, sino que contempla el papel definitivo de la subjetividad ante las nuevas tecnologías, por tanto, de la incertidumbre y la complejidad, dado que el conocimiento es un proceso humano y dinámico (lafrancesco, 2011; Núñez-Pérez, 2015).

Cabe destacar que el pensamiento, la subjetividad y el razonamiento crítico no son suficientes para la formación de un conocimiento que permita, a lo largo del proceso formativo del individuo, ser competente. Es por ello que se considera necesario implementar estrategias pedagógicas, con herramientas tecnológicas que permitan al estudiante interactuar en grupo, las cuales se aplican en áreas del conocimiento tan disímiles como ingeniería, administración y educación
(Parada-Hernández \& Suárez-Aguilar, 2014; PintoSalamanca, Sofroni-Esmeral, \& Jiménez, 2015; Vergara-Arrieta, 2015).

En la actualidad existen múltiples herramientas al alcance de la mano, las cuales se aplican en todos los niveles educativos. Algunas de estas herramientas o instrumentos facilitan el proceso de aprendizaje, a la vez que sirven de soporte tecnológico para efectuar la gestión del conocimiento (Lago-de Vergara, Gamoba-Suárez, \& Montes-Miranda, 2014; Montes-Miranda, 2013; Pérez-Ortega \& Romo-Morales, 2015). Entre ellas se encuentran los sistemas de gestión del conocimiento o Learning Management Systems, LMS por sus siglas en inglés, la internet, los simuladores, el software educativo y las bases de datos (Angarita-Velandia, Fernández-Morales, \& Duarte, 2014; Davis, 2015; León-Medina \& Torres-Barahona, 2016; MedinaMonterrosa \& Carvajalino-Slaghekke, 2014; TorresOrtiz, 2012).

Estas herramientas promueven la interacción efectiva con el conocimiento que suele ser interesante y facilita la colaboración entre los miembros de una institución. Un estudio realizado por Kowledge Management Research Report Consulting en 2000 (como se citó en Wilson, 2002), estableció que las tecnologías tienen un gran nivel de relevancia en el apoyo de la creación del conocimiento.

Al revisar qué ocurre en el contexto americano, se refleja que este proceso está estructurado de acuerdo al desarrollo económico de cada país (Romero-González, 2014). Es por ello que países como Estados Unidos y Canadá, en Norte América, llevan la pauta en la utilización de estas herramientas tecnológicas en las aulas de clase permitiendo al estudiante ampliar sus conocimientos en todo lo relacionado con las nuevas tecnologías.

Por su parte, Centroamérica, México y Puerto Rico llevan la iniciativa de implementar estrategias que fomenten la gestión del conocimiento a través 
de las nuevas tecnologías. En este sentido, es importante reconocer que el revuelo informático está generando grandes cambios en el modo de enseñar y de aprender en las instituciones educativas (García-Llorente, 2015).

Loanterior coincidecon lo planteado porel Instituto Internacional de Planeamiento de la Educación de Buenos Aires (IIPE-BA, 2000), el cual indica que, en las instituciones educativas de América Latina, para alcanzar una gestión de conocimiento exitosa entre docentes y estudiantes, se deben emplear herramientas de apoyo tecnológico para optimizar el aprendizaje tanto en la educación básica como en la universitaria. La sociedad actual se describe a través de una constante revolución científica y tecnológica, y de allí se explican los continuos cambios que experimenta en los valores y en la formación misma del conocimiento científico (Cabero-Almenara \& Llorente-Cejudo, 2015).

Dentro de algunas inferencias iniciales del presente artículo, se asume, por observaciones, que las organizaciones educativas presentan falta de: responsabilidad, compromiso, interés y motivación con las actividades de aprendizaje y la alta dependencia del docente con relación al qué, cómo y cuándo se aprende, lo cual indica poca autonomía y autorregulación en el proceso formativo. Esta situación, posiblemente, es causada en las organizaciones por la falta de innovación en las tareas que promueven el cambio, así como por la falta de implementar acciones que respondan en forma más acertada a las exigencias de la sociedad actual (Fierro-Ulloa, 2013).

El propósito del presente artículo es exponer una reflexión teórica orientada a analizar la Gestión Tecnológica del Conocimiento, GTC, como herramienta moderna para la gerencia de las organizaciones educativas. A continuación se presentan las estrategias para la GTC, a fin de proponer lineamientos que promuevan su correcta utilización en el contexto de las instituciones educativas.

\section{Marco teórico y metodología}

2.1 Estrategias de gestión tecnológica del conocimiento

La estrategia para cualquier institución es de gran importancia, ya sea pequeña, mediana o grande; cada una tendrá sus niveles gerenciales que ocupan situales estratégicos; adicionalmente, cada una tendrá que pensar la manera más idónea de aplicar estrategias de mercado que le permitan posicionarse y mantener un desarrollo sustentable y sostenible.

Según Roldán (2000), la estrategia "es la búsqueda deliberada por un plan de acción que desarrolle la ventaja competitiva de un negocio y la multiplique" (p. 189). En este sentido, la estrategia dentro de un plan de acción implica determinar dónde está la institución, adónde quiere llegar y cómo hará para llegar hasta allí. Además, como indica Allepuz-Ros (2000), la estrategia dentro de la gestión tecnológica del conocimiento "es un proceso de reflexión y de decisión sobre los objetivos y acciones de la organización" (p. 165). Por ello, se considera que no es más que una decisión sobre las metas, propósitos y plan de acción para prosperar en el entorno, luego de considerar un diagnóstico de la organización, el contexto y sus competidores.

En tal sentido, Bustelo-Ruesta (2000) refiere que esta estrategia de gestión amerita decisiones sobre tres aspectos:

(...) la selección de los clientes a los que se le va a servir, selección de la proposición de valor que se les proporcionará y selección de las capacidades que permitirán crear valor tanto a los clientes como a los inversionistas (p. 112).

La estrategia implica, entonces, elegir tomar decisiones y descartar otras asumiendo el compromiso de las capacidades necesarias para desarrollarlas. 
Resulta evidente que la estrategia de la Gestión Tecnológica del Conocimiento, GTC, debe ajustar los medios a los objetivos. Las acciones que se preparan no solamente son tareas necesarias, sino que, además, forman parte del programa mediante los cuales se crean capacidades de mejoramiento. Esto requiere seleccionar unos mercados atractivos y conseguir una posición competitiva favorable a los mismos.

Es pertinente considerar, dentro de los planteamientos que se hacen, que la estrategia de GTC fomenta la toma de decisiones, en la búsqueda de cumplir los objetivos que se trazan las organizaciones educativas, a través de acciones que sean capaces de mejorar no solo a la institución sino también al contexto al que pertenece, además de las habilidades sociales que pueden desarrollar sus miembros, especialmente los docentes.

\subsection{Metodología}

El estudio se califica como descriptivo al exponer un análisis reflexivo sobre el comportamiento de la gestión del conocimiento, que, de acuerdo a Hurtado-de Barrera (2010), busca exponer las características que describen un fenómeno en un entorno específico. El trabajo implicó una revisión documental-bibliográfica y analítica de algunos aspectos de la gestión del conocimiento. Se utilizaron fuentes de información bibliográfica producidas por organizaciones multilaterales.

Hurtado-de Barrera (2010), también expresa que "un estudio es transeccional cuando el investigador estudia el evento en un único momento del tiempo" (p. 102). La autora considera que un estudio transeccional transversal se plantea una única medición de todos los datos utilizables correspondientes al periodo establecido, una recolección en un solo momento, cuya finalidad consiste en describir las variables dentro de los grupos y subgrupos definidos.

\section{Resultados y discusión}

\subsection{Entornos colaborativos}

Para que la organización pueda desarrollar un entorno colaborativo, según Bueno-Campos (2000):

(...) es puntual analizar y evaluar el ambiente en todas y cada una de sus dimensiones (socio-cultural, económica, tecnológica y legal) a partir de la acumulación de determinadas expectativas a corto, medio y largo plazo, única forma de que la empresa pueda plantear con un cierto rigor su competitividad o grado de competencia con otras empresas en el mercado en el que actúa (p. 32).

Según Edvisson y Malone (1999), el entorno se ha caracterizado por tres propiedades fundamentales: la dinamicidad, que enuncia la importancia de la velocidad de los cambios, en relación a la comunicación; la discontinuidad, que es la forma en que los factores del entorno se vienen permitiendo estos últimos años; y la ambigüedad, en que se concretan las posibles soluciones o manifestaciones de los entornos colaborativos.

Por otra parte, Hitz (como se citó en Cornellá, 2000), define el entorno colaborativo como un espacio de procesos de aprendizaje que enfatiza el: "(...) esfuerzo cooperativo o de grupo entre los docentes y los estudiantes, la participación activa y la interacción por parte de estudiantes y profesores, y el conocimiento que emerge desde un activo diálogo entre los participantes compartiendo sus ideas e información" (p. 36).

En este contexto, Gros (2008) unifica los criterios en una definición que establece que los entornos colaborativos son una "metodología de enseñanza basada en la creencia de que el aprendizaje se incrementa cuando se desarrollan destrezas cooperativas para aprender y solucionar los 
problemas así como acciones educativas en las cuales se ven inmersos" (p. 91).

La postura del investigador concuerda con los señalamientos de Hitz (como se citó en Gros, 2008), quien asegura que el entorno colaborativo de la organización permitirá desarrollar otras dimensiones de acuerdo a su capacidad de manejar los diferentes factores que se involucran en los procesos de la GTC, considerando las mejores estrategias para llevar a cabo el plan de acción, donde se contempla un entorno continuamente en evolución, dinámico, cambiante y globalizado.

La construcción colaborativa da cuenta de un proceso de indagación, de formulación de preguntas que lleve a la adquisición de nuevos conocimientos, partiendo de la evolución de los propios conocimientos. Es así como se pretende que los docentes trabajen en función de entornos colaborativos que en general han venido siendo unos signos que muestran evidencias de grandes cambios ocurridos últimamente en cada una de las dimensiones de los factores observados. Las características de los cambios, en estos entornos tecnológicos, sociales y legales han ido definiendo esta época como la era del cambio.

Además, se busca que los docentes tengan en cuenta características como las que últimamente han impregnado a la mayoría de los entornos colaborativos, produciendo unos efectos en la organización educativa, que se pueden identificar como amenazas y oportunidades. Se entiende que las amenazas miden los impactos negativos; es decir, representan las amenazas a los objetivos de la organización. Por otra parte, las oportunidades, que se constituyen en efectos positivos, que, al aprovecharse, mejoran los resultados.

\subsection{Gestión de un sistema experto}

Cuando se habla de la gestión de un sistema de expertos, es necesario señalar la definición de Peña-Ayala (2006), según la cual "es un sistema computacional que adquiere conocimiento especializado en un campo específico para explotarlo mediante métodos de razonamiento que emulan el desempeño del experto humano en la solución de problemas" (p. 11). Infiere el autor que es necesario encontrar y seleccionar las interpretaciones correctas de la información y que estén en consistencia con los datos, considerando aquellas que la evidencia oriente a descartar.

En el mismo orden de ideas, Henczel (2000) indica que, por lo general, un sistema de expertos puede comprender un amplio conocimiento específico a partir de un campo de interés, así comola aplicación de técnicas de búsqueda. Además de la habilidad para deducir nuevos conocimientos a partir de los actuales y de las experiencias obtenidas durante su operación; incluye un procesamiento simbólico, la capacidad para explicar un razonamiento propio y el empleo de diferentes técnicas de solución de problemas.

De otro lado, Cornellá (2000) señala los atributos más relevantes de la gestión de un sistema de expertos, en los cuales se destaca la búsqueda del conocimiento y su replicación en nuevos conocimientos a partir de sí mismos. Esto significa que la GTC con sistema de expertos se constituye en factor clave para los procesos de tratamiento y reconstrucción de la información.

De acuerdo con lo anterior, se puede observar la complejidad que implican los procesos de construcción de conocimiento a todos los niveles y donde, por la misma naturaleza de los diferentes sistemas, se constituyen como dependientes uno del otro. En este sentido, señala García-Morales (2000) algunos de estos niveles, entre los cuales se tienen:

En primer lugar, se conoce a los sistemas de interpretación, con los que se llevan a cabo los análisis de datos que han sido observados, para identificar el significado que envuelve $y$, a partir de ellos, describir las situaciones reales que expresan los datos que han sido medidos. 
En segundo plano, están los sistemas de diagnosis, orientados a identificar los estados de errores extraídos de datos observados, como, por ejemplo, el diagnóstico que se realiza para detectar enfermedades que pudieran ser infecciosas.

Por otro lado, se encuentran los sistemas de monitorización, a través de los cuales se pueden interpretar señales y establecer medidas para emitir respuestas a dichas señales. En este grupo se observan, por ejemplo, los sistemas de alarmas que avisan cuando es necesario alguna intervención. En este tipo de sistemas, la respuesta debe realizarse en tiempo real a fin de evitar alarmas innecesarias que no respondan a la realidad y mantener los niveles de credibilidad.

En cuarto lugar, se observan los sistemas de reparación, los cuales se orientan a desarrollar y ejecutar programas para solventar situaciones que han sido diagnosticadas como problemas. Mediante este tipo de sistemas, se pueden depurar, planificar y ejecutar los errores encontrados, comenzando por construir una descripción inicial del conocimiento del alumno y, a partir de él, se elabora la diagnosis y se procede al mejoramiento del aprendizaje.

En quinto lugar, se encuentran los llamados sistemas de control, que tienen la finalidad de controlar los comportamientos de los sistemas de forma integral, y debido a esto, su función está dada por tener que realizar interpretaciones constantemente de las situaciones de forma repetitiva y, desde allí, poder predecir las acciones que pudieran tomarse, así como emitir una diagnosis sobre las posibles causas del problema. Tiene la capacidad de formular planes correctivos y realizar el monitoreo de la ejecución.

Cuando se analizan las diferentes posturas de los autores citados anteriormente, se comparte la posición de García-Morales (2000), quien destaca que los sistemas de expertos: son sistemas que generan información, en correspondencia con los datos que le sean proporcionados, además de contribuir a la consolidación del conocimiento, a partir de la búsqueda, análisis e interpretación de datos, sin dejar de considerar al humano como factor fundamental para el funcionamiento de estos sistemas de experto, por lo que es este el que gestiona cada uno de los procesos, niveles y sistemas que existan.

También es relevante que el diseño del modelo de un sistema de gestión se realice con un enfoque social, en que el ser humano sea agente importante de transformación y se evidencie en el desarrollo de sus capacidades, las cuales contribuyan al impacto positivo sobre su entorno (Del Río-Cortina, 2012; Ruiz-Malbarez, 2011; Vergara-Arrieta \& Carbal, 2014).

\subsection{Sistemas basados en conocimiento}

Los sistemas basados en conocimiento constituyen un paso adelante de los sistemas de información tradicional, al intentar representar funciones cognitivas del ser humano como el aprendizaje y el razonamiento. Este tipo de sistemas descansan en los aportes de la inteligencia artificial desde un punto de vista amplio, así como en la ingeniería del conocimiento en lo más concreto (Peña-Ayala, 2006).

Desde los planteamientos de Peña-Ayala (2006), el sistema basado "en conocimiento está orientado hacia la automatización del análisis de problemas, la búsqueda de soluciones, la toma de decisiones y el empleo de conocimiento especializado en un campo específico de aplicación" (p. XI). Es importante reseñar que, entre ellos, se encuentran los sistemas expertos, los cuales, como se indicó anteriormente, se encargan de la representación del conocimiento en busca de aprovecharlo para las acciones de diagnóstico, enseñanza y monitoreo.

En este sentido, Bustelo-Ruesta (2000) indica que la estructura de estos sistemas está conformada por los cimientos de conocimientos y medios de comunicación hombre-máquina. Además, estos 
sistemas procesan la información para soportar el proceso de decisiones a tomar, así como resguardar el conocimiento técnico, necesario para minimizar los errores humanos, además de automatizar las acciones que por lo general sean repetitivas, $y$, de esa manera, proceder a la transmisión de información en la organización, permitiendo la reducción del tiempo en la realización de las actividades minimizando los costos.

Adicionalmente, Jackson (1998) indica que estos sistemas trabajan con aquellos programas que se realizan haciendo explícito el conocimiento en ellos, que además contienen información específica de un algo concreto y que realizan una tarea relativa a este particular. Es decir, programas que maniobran con conocimiento codificado para solucionar problemas en un aspecto especializado que generalmente requiere de experiencia humana.

En complemento con lo planteado por Liao (2005), este sistema de conocimiento se trata de un sistema informático que termina realizando las tareas que un humano experto y profesional haría, partiendo de los requerimientos, conocimientos $y$ experiencias, que habitualmente son necesarios para ello, y actuando de una forma muy parecida a como lo haría un profesional en cualquier área o ámbito laboral, con las explicaciones y justificaciones pertinentes.

De lo anterior se deduce que un sistema de conocimiento es aquel que se basa en el conocimiento y está formado por bases de conocimiento; en esencia es un sistema informático que, dotado de una gran cantidad de información sobre conocimientos concretos y bien definidos, dispone también de una serie de mecanismos para poner a funcionar estos conocimientos almacenados, favoreciendo la resolución de problemas.

\subsection{Sistemas de recuperación de información}

Al respecto de los sistemas de recuperación de información, los cuales son útiles en la gestión tecnológica del conocimiento, el Diccionario Mac Millan de Tecnología de la Informática (como se citó en Martínez-Méndez, 2004) define el término como las "técnicas empleadas para almacenar y buscar grandes cantidades de datos y ponerlos a disposición de los usuarios" (p. 2).

Sin embargo, Meadow, Boyce y Kraft (2006) afirman que los procesos de recuperar toda información son: "una disciplina que involucra la localización de una determinada información dentro de un almacén de información o base de datos". (p. 88). En opinión de este autor, los procesos para recuperar la información están relacionados con lo que se conoce como selectividad, en el sentido que la información que se pueda extraer se realiza respetando algunos criterios selectivos.

Pérez-Carballo y Strzalkowski (como se citó en Martínez-Méndez, 2004) coinciden con los autores anteriores sosteniendo que estos son "una típica tarea de traer documentos relevantes desde un gran archivo, en respuesta a una pregunta formulada y ordenarlos de acuerdo con su relevancia" (p. 2). En igual opinión, Grossman y Frieder (2000) expresan que la recuperación de información se orienta a "encontrar documentos relevantes, no encontrar simples correspondencias a unos patrones de bits" (p. 56).

Lo anterior plantea que la recuperación de información evita el debido nivel de incertidumbre presente en la respuesta, contribuyendo a una búsqueda exitosa; de manera que la exactitud se convierte en el criterio clave, y el valor agregado llega a ser el grado en que se procede a satisfacer cualquier requerimiento de información.

En este sentido, se espera que en la recuperación de información dentro de las organizaciones, que resulten de trasladar la información a un lenguaje normalizado, los documentos respondan a los 
requerimientos solicitados. Indudablemente, se debe considerar una posible diferencia entre los requerimientos del sistema y la percepción de satisfacción que el usuario pueda tener. Los investigadores coinciden con los postulados de Pérez-Carballo y Strzalkowski (como se citó en Martínez-Méndez, 2004) destacando como aspecto importante el aspecto de predecir del usuario, en el sentido en que se debe desarrollar la intuición, así como los criterios seguidos para la construcción de la información contenida en los documentos y que sean desasociados de los sistemas de control.

\subsection{Minería de datos}

La minería de datos se usa en todo tipo de datos posibles, desde información numérica a imágenes satelitales, exámenes médicos y registros de computador, por mencionar algunos (TimaránPereira \& Yepes-Chamorro, en prensa). Tal como lo señala Bueno-Campos (1998), cualquier objeto compone en sí un dato, por lo cual la minería de datos posee muchísimas aplicaciones productivas como el marketing, manufactura, internet y análisis financiero, entre otros. Con tantos datos, se necesita limpiarlos y ordenarlos. Una vez efectuada esta tarea, se puede decir que se tiene la información. Esta tiene que ser manejada con un modelo para conseguir deducciones o conclusiones a los que se les llama conocimiento.

Bueno-Campos (1998) indica que no existe un modelo único y exclusivo de tratamiento de datos. Es por ello que el modelo a adoptar dependerá del contexto y las necesidades. Existen factores que deben ser tomados en cuenta, como lo son: la efectividad del modelo para generar buenas derivaciones, y si lo que de él resulta es lo requerido o es entendible para el ser humano.

Desde otra perspectiva, Pérez-López y SantínGonzález (2007) definen la minería de datos como el conjunto de técnicas y tecnologías que permiten explorar grandes bases de datos, de manera automática o semiautomática. Explica el autor que, básicamente, la minería de datos surge para ayudar a comprender el contenido de un repositorio de datos. Ahora bien, el conocimiento son datos analizados. Entonces, para este análisis hay distintas formas de minería de datos. En este sentido, un modelo es una manera de aplicar un procedimiento a un conjunto de datos para obtener información de los mismos. Esos datos deben ser organizados y pasados por un tratamiento mediante un algoritmo que da pie a que se consigan unos resultados.

Los autores de esta reflexión se identifican con la posición de Pérez-López y Santín-González (2007) cuando establecen que la minería de datos puede ser empleada con cualquier dato existente, ya que cualquier cosa está conformada por datos, y estos, a su vez, deben ser depurados y ordenados para lograr obtener lo que se conoce como información, generando así el tan anhelado conocimiento. De forma general, es importante que los miembros de las organizaciones comprendan que los datos son la materia prima bruta, y en el momento en que los usuarios les atribuyen algún significado especial pasan a convertirse en información.

\subsection{Recomendaciones para implementar la GTC}

La implementación de la GTC debería efectuarse a través de entornos que promuevan la interrelación de los miembros de la institución, permitiendo el intercambio de conocimiento. En este sentido, los ambientes colaborativos surgen como alternativa para la producción y difusión del conocimiento (Flores-Caicedo, 2010), junto con la utilización de expertos en algún conocimiento dela organización, que permita documentar información histórica de relevancia para la institución.

Igualmente, recomendable es el realizar una serie de procesos que incluyan lectura, escritura, conferencias, trabajo en equipo, sueños diarios o trabajo en un equipo directivo (Correa-Uribe, Rosero-Jiménez, \& Segura-Jiménez, 2008). Lo anterior, complementado con actividades grupales, permitirá potenciar las acciones 
colaborativas, de modo que los grupos de trabajo desarrollen procesos empáticos, para así afianzar las habilidades interpersonales en la institución. Por último, se recomienda desarrollar actividades de dinámicas entre los grupos de docentes, de manera que se consolide el conocimiento de las emociones que conduzcan a un mayor optimismo y motivación.

\section{Conclusiones}

La Gestión Tecnológica del Conocimiento, GTC, se constituye actualmente en una herramienta importante para la gerencia de las instituciones educativas de cualquier nivel de formación. Entre los hallazgos se tiene que las estrategias más empleadas para la gestión del conocimiento, aunque no muy estables, son: la utilización de espacios colaborativos, los sistemas de recuperación de información, la gestión y utilización de expertos, así como los sistemas basados en conocimiento; en contraste, son escasas las técnicas de gestión documental.

Para aprovechar los beneficios de la GTC en una organización, se recomienda desarrollar actividades que conduzcan a transformar el conocimiento a través de programas de socialización, mediante compartir experiencias e ideas personales. También será necesario llevar a cabo procesos de externalización, que implican el paso del conocimiento tácito colectivo al explícito; lo anterior complementado con el intercambio de conocimientos explícitos, documentos compartidos y la interiorización, en la que el conocimiento explícito colectivo se transforma en conocimiento tácito individual.

\section{Referencias}

Allepuz-Ros, M. (2000). La gestión del capital intelectual: nuevos parámetros de análisis para la economía de la información. Jornadas Españolas de Documentación, 7, 499-505.
Angarita-Velandia, M. A., Fernández-Morales, F. H., \& Duarte, J. E. (2014). La didáctica y su relación con el diseño de ambientes de aprendizaje: una mirada desde la enseñanza de la evolución de la tecnología. Revista de Investigación, Desarrollo e Innovación, 5 (1), 46-55. doi: 10.19053/20278306.3138

Bueno-Campos, E. (1998). El capital intangible como clave estratégica en la competencia actual. Boletín de Estudios Económicos, 53 (164), 207-229. Recuperado de: http://search.proquest.com/ope nview/3ab60647cc71baa198d764bab1a0ea34/1? pq-origsite $=$ gscholar

Bueno-Campos, E. (2000). De la sociedad de la información a la del conocimiento y el aprendizaje: La necesidad de programas de dirección del conocimiento y el aprendizaje. Jornadas Españolas de Documentación, 7, 647-657.

Bustelo-Ruesta, C. (2000). Gestión documental en las empresas: una aproximación práctica. Jornadas Españolas de Documentación, 7, 189-195.

Cabarcas-Álvarez, A. (2012). Cloud computing; tecnología verde como estrategia para la responsabilidad social empresarial. Saber, Ciencia y Libertad, 7 (2), 135-142. Recuperado de: http:// www.sabercienciaylibertad.com/ojs/index.php/ scyl/article/view/132/0

Cabero-Almenara, J., \& Llorente-Cejudo, M. del C. (2015). Tecnologías de la información y la comunicación (TIC): escenarios formativos y teorías del aprendizaje. Revista Lasallista de Investigación, 12 (2), 186-193. Recuperado de http://www.scielo. org.co/scielo.php?script=sci_arttext\&pid=S179444492015000200019\&lng=es\&tlng=es.

Castellanos-Niño, C. A. (2012). Interacción social en asesoría de proyectos escolares mediados por el e-Learning. Revista de Investigación, Desarrollo e Innovación, 2 (2), 30-38. Recuperado de: http://revistas.uptc.edu.co/revistas/index.php/ investigacion_duitama/article/view/1313 
Cornellá, A. (2000). La gestión de la información en las organizaciones. Quaderns Digitals. Recuperado de: http://www.quadernsdigitals.net/datos_web/ hemeroteca/r_1/nr_8/a_82/82.html

Correa-Uribe, G., Rosero-Jiménez, S. L., \& SeguraJiménez, H. (2008). Diseño de un modelo de gestión del conocimiento para la Escuela Interamericana de Bibliotecología. Revista Interamericana de Bibliotecología, 31 (1), 85-108. Recuperado de: http://www.scielo.org.co/scielo.php?script=sci_ arttext\&pid=S0120-09762008000100005\&lng=es \&tlng=es.

Davis, R. (2015). Bridge programs: a link between ESL and content-based courses. A case study: UEES. Saber, Ciencia y Libertad, 10 (1), 181-188. Recuperado de: https://dialnet.unirioja.es/servlet/ articulo?codigo $=5329114$

Del Río-Cortina, J. (2012). Desarrollo de actividades de emprendimiento con innovación y responsabilidad social en los hoteles en la ciudad de Cartagena de Indias. Saber, Ciencia y Libertad, 7 (2), 113-124. Recuperado de: https://dialnet. unirioja.es/servlet/articulo?codigo $=5109395$

Edvisson, L., \& Malone, M. (1999). El capital intelectual. Barcelona, España: Gestión 2000.

Fierro-Ulloa, I. (2013). Comportamiento organizacional positivo: implicaciones para la organización actual. Saber, Ciencia y Libertad, 8 (2), 103-111. Recuperado de: https://dialnet.unirioja. es/servlet/articulo?codigo $=5104984$

Flores-Caicedo, J. C. (2010). La gestión del conocimiento y las herramientas colaborativas: una alternativa de aplicación en instituciones de educación superior. Revista de Investigación, 34 (71), 11-31. Recuperado de: http://www.scielo. org.ve/scielo.php?script=sci_arttext\&pid=S101029142010000300002\&lng=es\&tlng=es.
Garcés-Pretel, M., Ruiz-Cantillo, R., \& MartínezÁvila, D. (2014). Transformación pedagógica mediada por tecnologías de la información y la comunicación (TIC). Saber, Ciencia y Libertad, 9 (2), 217-228. Recuperado de: https://dialnet.unirioja. es/descarga/articulo/5104968.pdf

García-Llorente, H. J. (2015). Multialfabetización en la sociedad del conocimiento: competencias informacionales en el sistema educativo. Revista Lasallista de Investigación, 12 (2), 225241. Recuperado de http://www.scielo.org. co/scielo.php?script $=$ sci_arttext $\&$ pid $=$ S179444492015000200023\&lng=es\&tlng=es.

García-Morales, E. (Noviembre de 2000). Herramientas para la gestión del conocimiento: el Mapa Documental, una experiencia práctica de implantación sobre la Intranet corporativa. En II Jornadas sobre Gestión del Conocimiento: "Claves para la implantación de programas de gestión del conocimiento". Evento llevado a cabo en Madrid, España.

Gros, B. (2008). Aprendizajes, conexiones y artefactos: "La producción colaborativa del conocimiento". Barcelona, España: Editorial Gedisa.

Grossman, D.A., \& Frieder, O. (1998). Information retrieval: algorithms and heuristics. Boston, Estados Unidos: Kluwer Academia Publishers.

Henczel, S. (2000). The information audit as the first step towards effective knowledge management. INSPEL, 34 (3), 210-226. Recuperado de: http:// forge.fh-potsdam.de/ IFLA/INSPEL/00-3hesu.pdf

Hurtado-de Barrera, J. (2010). Guía para la comprensión holística de la ciencia. Caracas, Venezuela: Fundación Sypal.

lafrancesco, G. M. (2011). Algunas problemáticas de la investigación y pedagogía en educación en Colombia: estrategias para enfrentarlas y resolverlas. Revista de Investigación, Desarrollo e 
Innovación, 1 (2), 7-16. Recuperado de: http:// revistas.uptc.edu.co/index.php/investigacion duitama/article/view/1298

Instituto Internacional de Planeamiento de la Educación Buenos Aires [IIPE-BA]. (2000). Desafíos de la educación. Diez módulos destinados a los responsables de los procesos de transformación educativa. Recuperado de: http://unesdoc.unesco. org/images/0015/001591/159155s.pdf

Jackson, P. (1998). Introduction to expert systems. (3th ed.) Reading, Estados Unidos: Addison-Wesley.

Lago-de Vergara, D. Gamoba-Suárez, A. A., \& Montes-Miranda, A. J. (2014). Calidad de la educación superior: un análisis de sus principales determinantes. Saber, Ciencia y Libertad, 9 (1), 157170. Recuperado de: https://dialnet.unirioja.es/ servlet/articulo?codigo $=5104971$

León-Medina, J. X., \& Torres-Barahona, E. A. (2016). Herramienta para el diseño de sistemas de posicionamiento tridimensional usados en fabricación digital. Revista de Investigación, Desarrollo e Innovación, 6 (2), 155-167. doi: 10.19053/20278306.4603

Liao, S.H. (2005). Expert system methodologies and applications - a decade review from 1995 to 2004. Expert Systems with Applications, 28 (1), 93103. doi: 10.1016/j.eswa.2004.08.003

Martínez-Méndez, F.J. (2004). Recuperación de información: modelos, sistemas y evaluación. Murcia, España: Kiosko JMC.

Meadow, C.T., Boyce, B.R., \& Kraft, D.H. (2006). Text information retrieval systems. (3th ed.). Bingley, Reino Unido: Emerald Group Publishing Limited.

Medina-Monterrosa, P., \& Carvajalino-Slaghekke, A. (2014). Ni al tigre ni al cuero: la toma de decisiones y el uso los sistemas de soporte y de ayuda a la decisión SSD-SAD. Saber, Ciencia y
Libertad, 9 (1), 115-128. Recuperado de: http:// www.sabercienciaylibertad.com/ojs/index.php/ scyl/article/view/26

Montes-Miranda, A. J. (2013). La educación básica en Colombia: una mirada a las políticas educativas. Saber, Ciencia y Libertad, 8 (2), 141-155. Recuperado de: http://www.sabercienciaylibertad.com/ojs/ index.php/scyl/article/view/106

Novoa-Ruiz, J. A. (2013). La cuestión integral del siglo XXI. Saber, Ciencia y Libertad, 8 (1), 71-83. Recuperado de: http://www.sabercienciaylibertad. com/ojs/index.php/scyl/article/view/116

Núñez-Pérez, V. (2015). Pedagogía social e interculturalismo: una lectura posible. Revista de Investigación, Desarrollo e Innovación, 5 (2), 141149. doi: $10.19053 / 20278306.3716$

Parada-Hernández, A., \& Suárez-Aguilar, Z.E. (2014). Influencia de las Tecnologías de la Información y la Comunicación en la apropiación de conceptos de electrónica análoga, en estudiantes de grado séptimo de educación básica. Revista de Investigación, Desarrollo e Innovación, 5 (1), 20-31. doi: $10.19053 / 20278306.3137$

Peña-Ayala, A. (2006). Sistemas basados en conocimiento: una base para su concepción y desarrollo. México, D.F., México: Instituto Politécnico Nacional.

Pérez-López, C., \& Santín-González, D. (2007). Minería de datos, técnicas y herramientas. Madrid, España: International Thompson Ediciones Paraninfo, S.A.

Pérez-Ortega, G., \& Romo-Morales, G. (2015). Liderazgo transformacional en los docentes universitarios desde la perspectiva estudiantil. Estudio de caso: Universidad Nacional de Colombia, sede Medellín. Saber, Ciencia y Libertad, $10(1), 203-218$. 
Pinto-Salamanca, M. L., Sofrony-Esmeral, J. I., \& Jiménez, D. F. (2015). Detección de colisiones con librerías V-Collide y PhysX para interacción virtual con interfaces hápticas. Revista de Investigación, Desarrollo e Innovación, 5 (2), 119-128. doi: 10.19053/20278306.3721

Roldán, J. L. (2000). Sistemas de información ejecutivos (EIS): Un estudio descriptivo sobre la situación en España. En M.J. Selva-Domínguez (Ed.): La empresa del siglo XXI: Finanzas, tecnologías y sistemas de información, Vol. II (pp. 237-254). Cádiz, España: Diputación de Cádiz Servicio de Publicaciones.

Romero-González, Z. (2014). El sistema de aseguramiento de la calidad en la educación superior. Saber, Ciencia y Libertad, 9 (2), 11-14. Recuperado de: http://sabercienciaylibertad.com/ ojs/index.php/scyl/article/view/2/0

Ruiz-Malvarez, M. C. (2011). La responsabilidad social empresarial y la obsolescencia programada. Saber, Ciencia y Libertad, 6 (1), 127-135. Recuperado de: https://dialnet.unirioja.es/servlet/ articulo?codigo $=3997367$

Timarán-Pereira, R., \& Yepes-Chamorro, M. C. (En prensa). Caracterización de la supervivencia de mujeres con cáncer invasivo de cuello uterino usando minería de datos. Revista de Investigación, Desarrollo e Innovación, 7 (1).
Torres-Ortiz, J. A. (2012). Incidencia de Moodle en las prácticas pedagógicas en modalidad educativa B-Learning. Revista de Investigación, Desarrollo e Innovación, 2 (2), 39-48. Recuperado de: http:// revistas.uptc.edu.co/index.php/investigacion_ duitama/article/view/1315

Torres-Ortiz, J. A., \& Duarte, J. E. (2016). Los procesos pedagógicos administrativos y los aspectos socio-culturales de inclusión y tecnopedagogía a través de las tendencias pedagógicas en educación a distancia y virtual. Revista de Investigación, Desarrollo e Innovación, 6 (2), 179190. doi: $10.19053 / 20278306.4606$

Vergara-Arrieta, J. J. (2015). Los sistemas de medición del desempeño estratégico frente a la competitividad y sustentabilidad. Saber, Ciencia y Libertad, 10 (2), 17-26. Recuperado de: https://dialnet.unirioja.es/servlet/ articulo?codigo $=5295019$

Vergara-Arrieta, J., \& Carbal, A. (2014). Diseño de un sistema de gestión en responsabilidad social empresarial para pequeños hoteles en la ciudad de Cartagena. Saber, Ciencia y Libertad, 9 (2), 91108. Recuperado de: http://sabercienciaylibertad. com/ojs/index.php/scyl/article/view/7

Wilson, T. D. (2002). The nonsense of knowledge management. Information research, 8 (1). Recuperado de: http://www.informationr.net/ir/81/paper144.html 